\title{
Measurement of Neutrino Oscillation with KamLAND: Evidence of Spectral Distortion
}

T. Araki, ${ }^{1}$ K. Eguchi, ${ }^{1}$ S. Enomoto, ${ }^{1}$ K. Furuno, ${ }^{1}$ K. Ichimura, ${ }^{1}$ H. Ikeda, ${ }^{1}$ K. Inoue,${ }^{1}$ K. Ishihara, ${ }^{1, *}$ T. Iwamoto, ${ }^{1,}$ T. Kawashima, ${ }^{1}$ Y. Kishimoto, ${ }^{1}$ M. Koga,${ }^{1}$ Y. Koseki, ${ }^{1}$ T. Maeda, ${ }^{1}$ T. Mitsui, ${ }^{1}$ M. Motoki, ${ }^{1}$ K. Nakajima, ${ }^{1}$ H. Ogawa, ${ }^{1}$ K. Owada, ${ }_{1}^{1}$ J.-S. Ricol, ${ }^{1}$ I. Shimizu, ${ }^{1}$ J. Shirai, ${ }^{1}$ F. Suekane,${ }^{1}$ A. Suzuki, ${ }^{1}$ K. Tada,${ }^{1}$ O. Tajima, ${ }^{1}$ K. Tamae, ${ }^{1}$ Y. Tsuda, ${ }^{1}$ H. Watanabe, ${ }^{1}$ J. Busenitz, ${ }^{2}$ T. Classen, ${ }^{2}$ Z. Djurcic, ${ }^{2}$ G. Keefer, ${ }^{2}$ K. McKinny, ${ }^{2}$ D.-M. Mei, ${ }^{2,+}$ A. Piepke, ${ }^{2}$ E. Yakushev, ${ }^{2}$ B. E. Berger, ${ }^{3}$ Y. D. Chan, ${ }^{3}$ M. P. Decowski, ${ }^{3}$ D. A. Dwyer, ${ }^{3}$ S. J. Freedman, ${ }^{3}$ Y. Fu, ${ }^{3}$ B. K. Fujikawa, ${ }^{3}$ J. Goldman, ${ }^{3}$ F. Gray, ${ }^{3}$ K. M. Heeger, ${ }^{3}$ K. T. Lesko, ${ }^{3}$ K.-B. Luk, ${ }^{3}$ H. Murayama, ${ }^{3,8}$ A. W. P. Poon, ${ }^{3}$ H. M. Steiner, ${ }^{3}$ L. A. Winslow, ${ }^{3}$ G. A. Horton-Smith, ${ }^{4, \|}$ C. Mauger, ${ }^{4}$ R. D. McKeown, ${ }^{4}$ P. Vogel, ${ }^{4}$ C. E. Lane, ${ }^{5}$ T. Miletic, ${ }^{5}$ P. W. Gorham, ${ }^{6}$ G. Guillian, ${ }^{6}$ J. G. Learned, ${ }^{6}$ J. Maricic, ${ }^{6}$ S. Matsuno, ${ }^{6}$ S. Pakvasa, ${ }^{6}$ S. Dazeley, ${ }^{7}$ S. Hatakeyama, ${ }^{7}$ A. Rojas, ${ }^{7}$ R. Svoboda, ${ }^{7}$ B. D. Dieterle, ${ }^{8}$ J. Detwiler, ${ }^{9}$ G. Gratta, ${ }^{9}$ K. Ishii, ${ }^{9}$ N. Tolich, ${ }^{9}$ Y. Uchida,,${ }^{9, \mathbb{I}}$ M. Batygov, ${ }^{10}$ W. Bugg, ${ }^{10}$ Y. Efremenko, ${ }^{10}$ Y. Kamyshkov, ${ }^{10}$ A. Kozlov, ${ }^{10}$ Y. Nakamura, ${ }^{10}$ C. R. Gould, ${ }^{11}$ H. J. Karwowski, ${ }^{11}$ D. M. Markoff, ${ }^{11}$ J. A. Messimore, ${ }^{11}$ K. Nakamura, ${ }^{11}$ R. M. Rohm, ${ }^{11}$ W. Tornow, ${ }^{11}$ R. Wendell, ${ }^{11}$ A. R. Young, ${ }^{11}$ M.-J. Chen, ${ }^{12}$ Y.-F. Wang, ${ }^{12}$ and F. Piquemal ${ }^{13}$

\section{(KamLAND Collaboration)}

\author{
${ }^{1}$ Research Center for Neutrino Science, Tohoku University, Sendai 980-8578, Japan \\ ${ }^{2}$ Department of Physics and Astronomy, University of Alabama, Tuscaloosa, Alabama 35487, USA \\ ${ }^{3}$ Physics Department, University of California at Berkeley, Berkeley, California 94720, USA, \\ and Lawrence Berkeley National Laboratory, Berkeley, California 94720, USA \\ ${ }^{4}$ W. K. Kellogg Radiation Laboratory, California Institute of Technology, Pasadena, California 91125, USA \\ ${ }_{5}$ Physics Department, Drexel University, Philadelphia, Pennsylvania 19104, USA \\ ${ }^{6}$ Department of Physics and Astronomy, University of Hawaii at Manoa, Honolulu, Hawaii 96822, USA \\ ${ }^{7}$ Department of Physics and Astronomy, Louisiana State University, Baton Rouge, Louisiana 70803, USA \\ ${ }^{8}$ Physics Department, University of New Mexico, Albuquerque, New Mexico 87131, USA \\ ${ }^{9}$ Physics Department, Stanford University, Stanford, California 94305, USA \\ ${ }^{10}$ Department of Physics and Astronomy, University of Tennessee, Knoxville, Tennessee 37996, USA \\ ${ }^{11}$ Triangle Universities Nuclear Laboratory, Durham, North Carolina 27708, USA, \\ and Physics Departments at Duke University, North Carolina State University, \\ and the University of North Carolina at Chapel Hill, Chapel Hill, North Carolina, USA \\ ${ }^{12}$ Institute of High Energy Physics, Beijing 100039, People's Republic of China \\ ${ }^{13}$ CEN Bordeaux-Gradignan, IN2P3-CNRS and University Bordeaux I, F-33175 Gradignan Cedex, France
} (Received 15 June 2004; published 1 March 2005)

\begin{abstract}
We present results of a study of neutrino oscillation based on a 766 ton/year exposure of KamLAND to reactor antineutrinos. We observe $258 \bar{\nu}_{e}$ candidate events with energies above $3.4 \mathrm{MeV}$ compared to $365.2 \pm 23.7$ events expected in the absence of neutrino oscillation. Accounting for $17.8 \pm 7.3$ expected background events, the statistical significance for reactor $\bar{\nu}_{e}$ disappearance is $99.998 \%$. The observed energy spectrum disagrees with the expected spectral shape in the absence of neutrino oscillation at $99.6 \%$ significance and prefers the distortion expected from $\bar{\nu}_{e}$ oscillation effects. A two-neutrino oscillation analysis of the KamLAND data gives $\Delta m^{2}=7.9_{-0.5}^{+0.6} \times 10^{-5} \mathrm{eV}^{2}$. A global analysis of data from KamLAND and solar-neutrino experiments yields $\Delta m^{2}=7.9_{-0.5}^{+0.6} \times 10^{-5} \mathrm{eV}^{2}$ and $\tan ^{2} \theta=0.40_{-0.07}^{+0.10}$, the most precise determination to date.
\end{abstract}

DOI: 10.1103/PhysRevLett.94.081801

PACS numbers: $14.60 . \mathrm{Pq}, 26.65 .+\mathrm{t}, 28.50 . \mathrm{Hw}$

The first measurement of reactor antineutrino disappearance by KamLAND [1] suggested that solar-neutrino flavor transformation through the Mikheyev-SmirnovWolfenstein (MSW) [2] matter effect has a direct correspondence to antineutrino oscillation in a vacuum. Assuming CPT invariance, KamLAND and solar-neutrino experiments have restricted the solar oscillation parameters, eliminating all but the large-mixing-angle (LMAMSW) solution. This Letter reports more stringent constraints on neutrino oscillation parameters from KamLAND based on a 3 times longer exposure and a
$33 \%$ increase in the fiducial volume. Large variations in the reactor power production in Japan in 2003 allowed us to study the $\bar{\nu}_{e}$ flux dependence. The first evidence for spectral distortion in the $\bar{\nu}_{e}$ spectrum is provided here; spectral distortion is direct evidence of an oscillation effect.

KamLAND consists of 1 kton of ultrapure liquid scintillator (LS) contained in a 13-m-diameter transparent nylon-based balloon suspended in nonscintillating oil. The balloon is surrounded by 1879 photomultiplier tubes (PMTs) mounted on the inner surface of an 18-m-diameter 
spherical stainless steel vessel. Electron antineutrinos are detected via inverse $\beta$ decay, $\bar{\nu}_{e}+p \rightarrow e^{+}+n$, with a $1.8 \mathrm{MeV} \bar{\nu}_{e}$ energy threshold. The prompt scintillation light from the $e^{+}$gives an estimate of the incident $\bar{\nu}_{e}$ energy, $E_{\bar{\nu}_{e}}=E_{\text {prompt }}+\bar{E}_{n}+0.8 \mathrm{MeV}$, where $E_{\text {prompt }}$ is the prompt event energy including the positron kinetic energy and the annihilation energy, and $\bar{E}_{n}$ is the average neutron recoil energy, which is small. The $\sim 200 \mu$ s delayed 2.2 MeV $\gamma$ ray from neutron capture on hydrogen is a powerful tool for reducing background. On average, neutrons are captured within $9 \mathrm{~cm}$ and the spatial correlation between prompt and delayed signals is dominated by the vertex position resolution and capture $\gamma$ ray absorption length. A 3.2 kton water-Cherenkov detector surrounds the containment sphere, absorbing $\gamma$ rays and neutrons from the surrounding rock and tagging cosmic-ray muons. This outer detector (OD) is over $92 \%$ efficient for muons passing through the fiducial volume.

KamLAND is surrounded by 53 Japanese power reactor units. The reactor operation data, including thermal power generation, fuel burn up, and exchange and enrichment records, are provided by all Japanese power reactors and are used to calculate fission rates of each isotope. The averaged relative fission yields for the run period were ${ }^{235} \mathrm{U}:{ }^{238} \mathrm{U}:{ }^{239} \mathrm{Pu}:{ }^{241} \mathrm{Pu}=0.563: 0.079: 0.301: 0.057$. The expected $\bar{\nu}_{e}$ flux is calculated from the fission rates using the $\bar{\nu}_{e}$ spectra from Ref. [3]. The $\bar{\nu}_{e}$ contribution from Japanese research reactors and reactors outside of Japan is $4.5 \%$. We assume that these reactors have the same average fuel composition as the Japanese power reactors. The integrated thermal power flux over the detector live time was $701 \mathrm{~J} / \mathrm{cm}^{2}$.

We report on data collected between 9 March 2002 and 11 January 2004, including reanalysis of the data used in Ref. [1]. The central detector PMT array was upgraded on 27 February 2003 by commissioning 554 20-inch PMTs, increasing the photocathode coverage from $22 \%$ to $34 \%$, and improving the energy resolution from $7.3 \% / \sqrt{E(\mathrm{MeV})}$ to $6.2 \% / \sqrt{E(\mathrm{MeV})}$. The trigger threshold of 200 hit 17 inch PMTs corresponds to about $0.7 \mathrm{MeV}$ at the detector center. The trigger has an efficiency close to $100 \%$ above the interaction threshold. We use a prompt event energy analysis threshold of $2.6 \mathrm{MeV}$ to avoid backgrounds including the effect of antineutrinos from uranium and thorium decaying in the Earth (geoneutrinos).

The location of interactions inside the detector is determined from PMT hit timing; the energy is obtained from the number of observed photoelectrons after correcting for position and gain variations. Position and time dependence of the energy estimation are monitored periodically with $\gamma$-ray and neutron sources along the central vertical axis ( $\mathrm{z}$ axis) of the scintillator volume. Trace radioisotopes on the balloon and in the scintillator are also exploited. The systematic uncertainty in the energy scale at the $2.6 \mathrm{MeV}$ prompt event energy $\left(E_{\bar{\nu}_{e}} \simeq 3.4 \mathrm{MeV}\right)$ analysis threshold is
$2.0 \%$, corresponding to a $2.3 \%$ uncertainty in the number of events in an unoscillated reactor $\bar{\nu}_{e}$ spectrum.

The radial fiducial volume cut is relaxed from five [1] to $5.5 \mathrm{~m}$ in the present analysis, expanding the fiducial mass to 543.7 tons $\left(4.61 \times 10^{31}\right.$ free target protons). The radial positions of the prompt and delayed event are both required to be less than $5.5 \mathrm{~m}$. The $1.2 \mathrm{~m}$ cylindrical cut along the $\mathrm{z}$ axis previously used to exclude low energy backgrounds from thermometers is not applied. The event selection cuts for the time difference $(\Delta T)$ and position difference $(\Delta R)$ between the positron and delayed neutron are $0.5 \mu \mathrm{s}<$ $\Delta T<1000 \mu \mathrm{s}$ and $\Delta R<2 \mathrm{~m}$, respectively. The event energies are required to be $2.6 \mathrm{MeV}<E_{\text {prompt }}<$ $8.5 \mathrm{MeV}$ and $1.8 \mathrm{MeV}<E_{\text {delayed }}<2.6 \mathrm{MeV}$. The efficiency of all cuts is $(89.8 \pm 1.5) \%$.

The total volume of the liquid scintillator is $1171 \pm$ $25 \mathrm{~m}^{3}$, as measured by flow meters during detector filling. The nominal 5.5 -m-radius fiducial volume $\left(\frac{4}{3} \pi R^{3}\right)$ corresponds to $0.595 \pm 0.013$ of the total LS volume. The effective fiducial volume is defined by the cuts on the radial positions of the reconstructed event vertices. At present, only z-axis calibrations are available, so we assess the systematic uncertainty in the fiducial volume by studying uniformly distributed muon spallation products, identified as delayed coincidences following muons. We measure the position distribution of the $\beta$ decays of ${ }^{12} \mathrm{~B}(Q=$ $\left.13.4 \mathrm{MeV}, \tau_{1 / 2}=20.2 \mathrm{~ms}\right)$ and ${ }^{12} \mathrm{~N}(Q=17.3 \mathrm{MeV}$, $\tau_{1 / 2}=11.0 \mathrm{~ms}$ ), which are produced at the rate of about $60{ }^{12} \mathrm{~B} /{ }^{12} \mathrm{~N}$ events/kton/day. Fits to the energy distribution of these events indicate that the sample is mostly ${ }^{12} \mathrm{~B}$; the relative contribution of ${ }^{12} \mathrm{~N}$ is only $\sim 1 \%$. The number of ${ }^{12} \mathrm{~B} /{ }^{12} \mathrm{~N}$ events reconstructed in the fiducial volume compared to the total number in the entire LS volume is $0.607 \pm 0.006$ (stat) \pm 0.006 (syst). As a consistency check, in a similar study of spallation neutrons we find the ratio $0.587 \pm 0.013$ (stat).

The ${ }^{12} \mathrm{~B} /{ }^{12} \mathrm{~N}$ events typically have higher energy than $\bar{\nu}_{e}$ candidates, so an additional systematic error accounts for possible dependence of effective fiducial volume on energy. We constrain the variation to $2.7 \%$ by comparing the prompt and delayed event positions of delayed-neutron $\beta$ decays of ${ }^{9} \mathrm{Li}\left(Q=13.6 \mathrm{MeV}, \tau_{1 / 2}=178 \mathrm{~ms}\right)$ and ${ }^{8} \mathrm{He}\left(Q=10.7 \mathrm{MeV}, \tau_{1 / 2}=119 \mathrm{~ms}\right)$. The observed capture distance variation is a measure of the energy uniformity of the vertex finding algorithm. Combining the errors from the LS volume measurements, the ${ }^{12} \mathrm{~B} /{ }^{12} \mathrm{~N}$ volume ratio calibration, and the constraints on energy dependence, we obtain a $4.7 \%$ systematic error on the fiducial volume.

The rate of accidental coincidences increases in the outer region of the fiducial volume, since most background sources are external to the liquid scintillator. This background is estimated with a $10 \mathrm{~ms}$ to $20 \mathrm{~s}$ delayedcoincidence window and by pairing random singles events. 
These consistent methods predict $2.69 \pm 0.02$ events above the $2.6 \mathrm{MeV}$ threshold.

Above $2.6 \mathrm{MeV}$, neutrons and long-lived delayedneutron $\beta$ emitters are sources of correlated backgrounds. The $\sim 3000$ spallation-produced neutrons/kton/day are effectively eliminated with a $2 \mathrm{~ms}$ veto of the entire detector following a detected muon. The remaining fast neutrons come from muons missed by the OD or interacting in the rock just outside it. This background is reduced significantly by the OD and several layers of absorbers: the OD itself, the $2.5 \mathrm{~m}$ of nonscintillating oil surrounding the LS, and the $1 \mathrm{~m}$ of LS outside the fiducial volume. We estimate this background contributes fewer than 0.89 events to the data sample.

The uncorrelated background from ${ }^{12} \mathrm{~B} /{ }^{12} \mathrm{~N}$ spallation products is effectively suppressed by the delayedcoincidence requirement. However, the $\sim 1.5$ events/kton/ day in the delayed-neutron branches of ${ }^{9} \mathrm{Li}$ and ${ }^{8} \mathrm{He}$ mimic the $\bar{\nu}_{e}$ signal. From fits to the decay time and $\beta$-energy spectra we see mostly ${ }^{9} \mathrm{Li}$ decays; the contribution of ${ }^{8} \mathrm{He}$ relative to ${ }^{9} \mathrm{Li}$ is less than $15 \%$ at $90 \%$ C.L. For isolated, well tracked muons passing through the detector, we apply a $2 \mathrm{~s}$ veto within a $3 \mathrm{~m}$ radius cylinder around the track. We veto the entire volume for $2 \mathrm{~s}$ after one in $\sim 30$ muons, those that produce more than $\sim 10^{6}$ photoelectrons above minimum ionization or muons tracked with poor reliability. We estimate that $4.8 \pm 0.9{ }^{9} \mathrm{Li} /{ }^{8} \mathrm{He}$ events remain after the cuts. The dead time introduced by all muon cuts is $(9.7 \pm 0.1) \%$; the total live time including spallation cuts is $(515.1 \pm 0.3)$ days.

A third source of correlated background comes indirectly from the $\alpha$ decays of the radon daughter ${ }^{210} \mathrm{Po}$ in the liquid scintillator. The signal of the $5.3 \mathrm{MeV} \alpha$ particle is quenched below the threshold, but the secondary reaction ${ }^{13} \mathrm{C}(\alpha, n){ }^{16} \mathrm{O}$ produces events above $2.6 \mathrm{MeV}$. Special runs to observe the decay of ${ }^{210} \mathrm{Po}$ establish that there were $(1.47 \pm 0.20) \times 10^{9} \alpha$ decays during the live time of data taking. Using the ${ }^{13} \mathrm{C}(\alpha, n)$ reaction cross sections from Ref. [4], Monte Carlo simulations, and detailed studies of quenching effects to convert the outgoing neutron energy spectrum into a visible energy spectrum, we expect $10.3 \pm$ 7.1 events above $2.6 \mathrm{MeV}$. The spectrum exhibits two peaks near 6 and $4.4 \mathrm{MeV}$, from decays of levels in ${ }^{16} \mathrm{O}$ and from $\gamma$ decays following neutron inelastic scattering on ${ }^{12} \mathrm{C}$, respectively. The observed energy from neutronproton elastic scattering is mostly quenched below 2.6 MeV. This $\alpha$-induced background was not considered

TABLE I. Estimated systematic uncertainties (\%).

\begin{tabular}{lccc}
\hline \hline Fiducial volume & 4.7 & Reactor power & 2.1 \\
Energy threshold & 2.3 & Fuel composition & 1.0 \\
Efficiency of cuts & 1.6 & $\bar{\nu}_{e}$ spectra [3] & 2.5 \\
Live time & 0.06 & Cross section [5] & 0.2 \\
\hline Total systematic uncertainty & & & 6.5 \\
\hline \hline
\end{tabular}

in Ref. [1] and would have contributed $1.9 \pm 1.3$ additional background events ( $2.8 \pm 1.7$ total background events). The total background to the $\bar{\nu}_{e}$ signal above $2.6 \mathrm{MeV}$ in the present analysis is $17.8 \pm 7.3$ events, where the bound on the fast neutron background is accounted for in the uncertainty.

In the absence of antineutrino disappearance, we expect to observe $365.2 \pm 23.7$ (syst) $\bar{\nu}_{e}$ events above $2.6 \mathrm{MeV}$, where the systematic uncertainty is detailed in Table I. We observe 258 events, confirming $\bar{\nu}_{e}$ disappearance at the 99.998\% significance level. Assuming Gaussian statistics, a $4 \sigma$ deviation would be needed to explain this deficit. The average $\bar{\nu}_{e}$ survival probability is $0.658 \pm 0.044$ (stat) \pm 0.047 (syst), where the background error has been included in the systematic uncertainty. The effective baseline varies with power output of the reactor sources involved, so the survival probabilities for different periods are not directly comparable. Applying the new analysis on the previously reported data [1] gives $0.601 \pm 0.069$ (stat) \pm 0.042 (syst), in agreement with $0.589 \pm 0.085$ (stat) \pm 0.042 (syst), after correction for the $(\alpha, n)$ background.

After September 2002, a number of Japanese nuclear reactors were off, as indicated in Fig. 1(a). This decreased
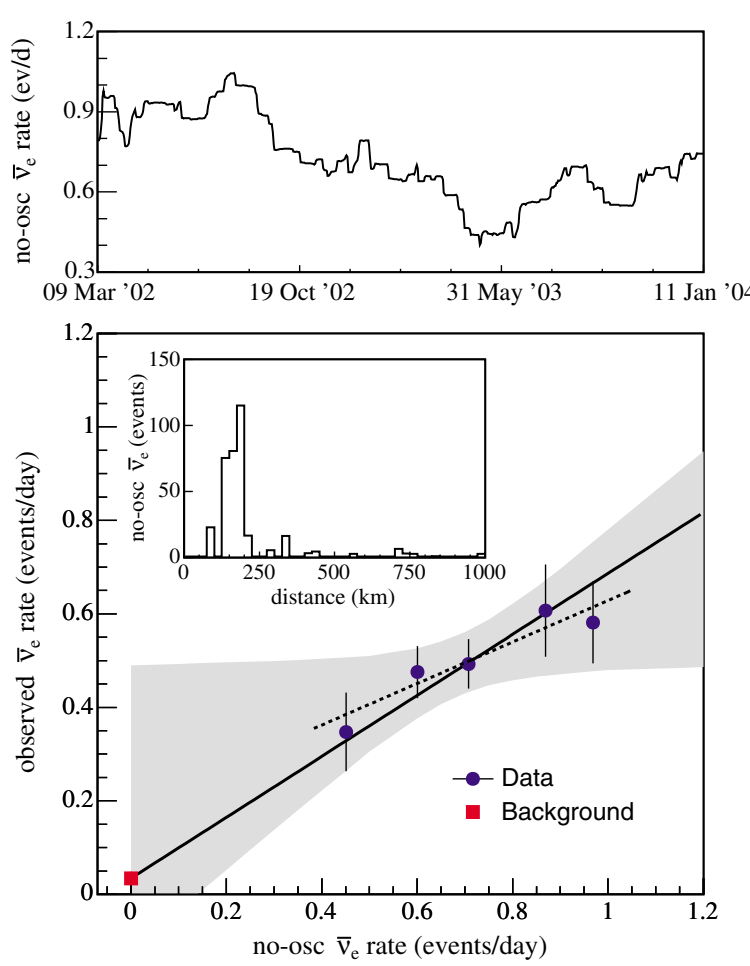

FIG. 1 (color). (a) Estimated time variation of the reactor $\bar{\nu}_{e}$ flux at KamLAND assuming no antineutrino oscillation. (b) Observed $\bar{\nu}_{e}$ event rate versus no-oscillation reactor $\bar{\nu}_{e}$ flux. Data points correspond to intervals of approximately equal $\bar{\nu}_{e}$ flux. The dashed line is a fit; the $90 \%$ C.L. is shown in gray. The solid line is a fit constrained to the expected background. The reactor distance distribution for $\bar{\nu}_{e}$ events in the absence of oscillations is shown in the inset. 
the expected no-oscillation $\bar{\nu}_{e}$ flux by more than a factor of 2. In Fig. 1(b) the signal counts are plotted in bins of approximately equal $\bar{\nu}_{e}$ flux corresponding to total reactor power. For $\Delta m^{2}$ and $\tan ^{2} \theta$ determined below and the known distributions of reactor power level and distance, the expected oscillated $\bar{\nu}_{e}$ rate is well approximated by a straight line. The slope can be interpreted as the $\bar{\nu}_{e}$ rate suppression factor and the intercept as the reactorindependent constant background rate. Figure 1(b) shows the linear fit and its $90 \%$ C.L. region. The intercept is consistent with known backgrounds, but substantially larger backgrounds cannot be excluded; hence this fit does not usefully constrain speculative sources of antineutrinos such as a nuclear reactor at the Earth's core [6]. The predicted KamLAND rate for typical 3 TW geo-reactor scenarios is comparable to the expected $17.8 \pm 7.3$ event background and would have minimal impact on the analysis of the reactor power dependence signal. In the following we consider contributions only from known antineutrino sources.

Figure 2(a) shows the correlation of the prompt and delayed event energy after all selection cuts except for the $E_{\text {delayed }}$ cut. The prompt energy spectrum above 2.6 MeV is shown in Fig. 2(b). The data evaluation method with an unbinned maximum likelihood fit to two-flavor neutrino oscillation is similar to the method used previously [1]. In the present analysis, we account for the ${ }^{9} \mathrm{Li}$, accidental, and the ${ }^{13} \mathrm{C}(\alpha, n){ }^{16} \mathrm{O}$, background, rates. For the $(\alpha, n)$ background, the contribution around $6 \mathrm{MeV}$ is allowed to float because of uncertainty in the cross section,

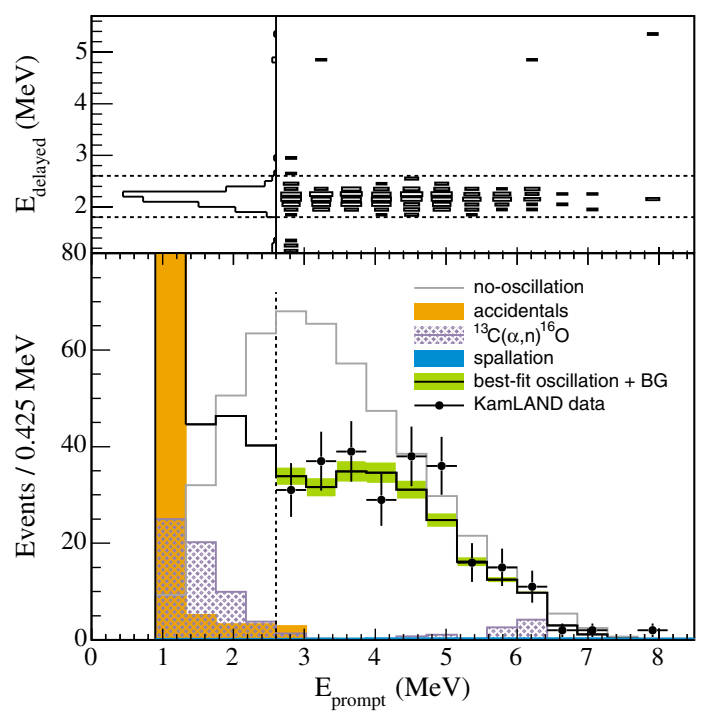

FIG. 2 (color). (a) The correlation between the prompt and delayed event energies after cuts. The three events with $E_{\text {delayed }} \sim 5 \mathrm{MeV}$ are consistent with neutron capture on carbon. (b) Prompt event energy spectrum of $\bar{\nu}_{e}$ candidate events with associated background spectra. The shaded band indicates the systematic error in the best-fit reactor spectrum above $2.6 \mathrm{MeV}$. The first bin in the accidentals histogram contains $\sim 113$ events. while the contributions around 2.6 and $4.4 \mathrm{MeV}$ are constrained to within $32 \%$ of the estimated rate. We allow for a $10 \%$ energy scale uncertainty for the $2.6 \mathrm{MeV}$ contribution due to neutron quenching uncertainty. The best-fit spectrum together with the backgrounds is shown in Fig. 2(b); the best fit for the rate-and-shape analysis is $\Delta m^{2}=$ $7.9_{-0.5}^{+0.6} \times 10^{-5} \mathrm{eV}^{2}$ and $\tan ^{2} \theta=0.46$, with a large uncertainty on $\tan ^{2} \theta$. A shape-only analysis gives $\Delta m^{2}=(8.0 \pm$ $0.5) \times 10^{-5} \mathrm{eV}^{2}$ and $\tan ^{2} \theta=0.76$.

Taking account of the backgrounds, the Baker-Cousins $\chi^{2}$ for the best fit is 13.1 (11 d.o.f.). To test the goodness of fit we follow the statistical techniques in Ref. [7]. First, the data are fit to a hypothesis to find the best-fit parameters. Next, we bin the energy spectrum of the data into 20 equalprobability bins and calculate the Pearson $\chi^{2}$ statistic $\left(\chi_{p}^{2}\right)$ for the data. Based on the particular hypothesis 10000 spectra were generated using the parameters obtained from the data and $\chi_{p}^{2}$ was determined for each spectrum. The confidence level of the data is the fraction of simulated spectra with a higher $\chi_{p}^{2}$. For the best-fit oscillation parameters and the a priori choice of 20 bins, the goodness of fit is $11.1 \%$ with $\chi_{\mathrm{p}}^{2} /$ d.o.f. $=24.2 / 17$. The goodness of fit of the scaled no-oscillation spectrum where the normalization was fit to the data is $0.4 \%\left(\chi_{\mathrm{p}}^{2} /\right.$ d.o.f. $\left.=37.3 / 18\right)$. We note that the $\chi_{p}^{2}$ and goodness-of-fit results are sensitive to the choice of binning.

To illustrate oscillatory behavior of the data, we plot in Fig. 3 the $L_{0} / E$ distribution, where the data and the best-fit spectra are divided by the expected no-oscillation spectrum. Two alternative hypotheses for neutrino disappearance, neutrino decay [8] and decoherence [9], give different $L_{0} / E$ dependences. As in the oscillation analysis, we survey the parameter spaces and find the best-fit points

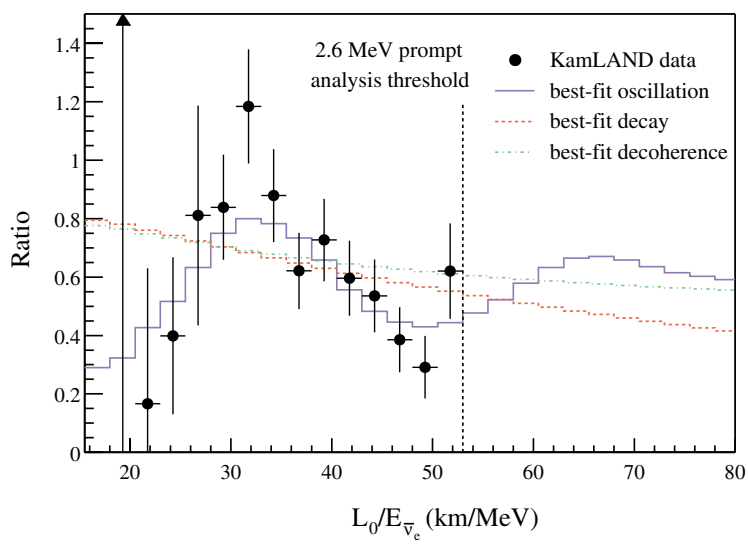

FIG. 3 (color). Ratio of the observed $\bar{\nu}_{e}$ spectrum to the expectation for no-oscillation versus $L_{0} / E$. The curves show the expectation for the best-fit oscillation, best-fit decay, and best-fit decoherence models taking into account the individual time-dependent flux variations of all reactors and detector effects. The data points and models are plotted with $L_{0}=180 \mathrm{~km}$, as if all antineutrinos detected in KamLAND were due to a single reactor at this distance. 

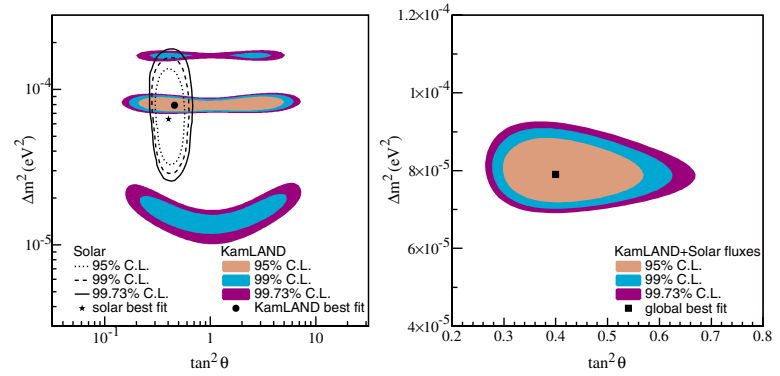

FIG. 4 (color). (a) Neutrino oscillation parameter allowed region from KamLAND antineutrino data (shaded regions) and solar-neutrino experiments (lines) [13]. (b) Result of a combined two-neutrino oscillation analysis of KamLAND and observed solar-neutrino fluxes under the assumption of $C P T$ invariance. The fit gives $\Delta m^{2}=7.9_{-0.5}^{+0.6} \times 10^{-5} \mathrm{eV}^{2}$ and $\tan ^{2} \theta=0.40_{-0.07}^{+0.10}$ including the allowed 1-sigma parameter range.

at $\left(\sin ^{2} \theta, m / c \tau\right)=(1.0,0.011 \mathrm{MeV} / \mathrm{km})$ for decay and $\left(\sin ^{2} 2 \theta, \gamma^{0}\right)=(1.0,0.030 \mathrm{MeV} / \mathrm{km})$ for decoherence, using the notation of the references. Applying the goodnessof-fit procedure described above, we find that decay has a goodness of fit of only $0.7 \%\left(\chi_{\mathrm{p}}^{2} /\right.$ d.o.f. $\left.=35.8 / 17\right)$, while decoherence has a goodness of fit of $1.8 \%\left(\chi_{\mathrm{p}}^{2} /\right.$ d.o.f. $=$ $32.2 / 17)$. We note that, while the present best-fit neutrino decay point has already been ruled out by solar-neutrino data [10] and observation of SN1987A [11], the decay model is used here as an example of a scenario resulting in a $\bar{\nu}_{e}$ deficit. If we do not assume $C P T$ invariance and allow the range $0.5<\sin ^{2} \theta<0.75$, then the decay scenario considered here can avoid conflict with solarneutrino and SN1987A data.

The allowed region contours in $\Delta m^{2}-\tan ^{2} \theta$ parameter space derived from the $\Delta \chi^{2}$ values (e.g., $\Delta \chi^{2}<5.99$ for 95\% C.L.) are shown in Fig. 4(a). The best-fit point is in the region commonly characterized as LMA I. Maximal mixing for values of $\Delta m^{2}$ consistent with LMA I is allowed at the $62.1 \%$ C.L. Because of distortions in the spectrum, the LMA II region (at $\Delta m^{2} \sim 2 \times 10^{-4} \mathrm{eV}^{2}$ ) is disfavored at the $98.0 \%$ C.L., as are larger values of $\Delta m^{2}$ previously allowed by KamLAND. The allowed region at lower $\Delta m^{2}$ is disfavored at the $97.5 \%$ C.L., but this region is not consistent with the LMA region determined from solarneutrino experiments assuming $C P T$ invariance.

A two-flavor analysis of the KamLAND data and the observed solar-neutrino fluxes [12], with the assumption of $C P T$ invariance, restricts the allowed $\Delta m^{2}-\tan ^{2} \theta$ parameters as shown in Fig. 4(b). The sensitivity in $\Delta m^{2}$ is dominated by the observed distortion in the KamLAND spectrum, while solar-neutrino data provide the best constraint on $\theta$. The combined analysis gives $\Delta m^{2}=$ 7.9 ${ }_{-0.5}^{+0.6} \times 10^{-5} \mathrm{eV}^{2}$ and $\tan ^{2} \theta=0.40_{-0.07}^{+0.10}$.

The conclusion that the LMA II region is excluded is strengthened by the present result. The observed distortion of the spectral shape supports the conclusion that the observation of reactor $\bar{\nu}_{e}$ disappearance is due to neutrino oscillation. Statistical uncertainties in the KamLAND data are now on the same level as systematic uncertainties. Current efforts to perform full-volume source calibrations and a reevaluation of reactor power uncertainties should reduce the systematic uncertainties.

The KamLAND experiment is supported by the COE program under grant no. 09CE2003 of the Japanese Ministry of Education, Culture, Sports, Science, and Technology, and under the United States Department of Energy grant no. DEFG03-00ER41138. The reactor data are provided by courtesy of the following electric associations in Japan: Hokkaido, Tohoku, Tokyo, Hokuriku, Chubu, Kansai, Chugoku, Shikoku, and Kyushu Electric Power Companies, Japan Atomic Power Co., and Japan Nuclear Cycle Development Institute. The Kamioka Mining and Smelting Company has provided service for activities in the mine.

*Present address: ICRR, University of Tokyo, Gifu, Japan †Present address: ICEPP, University of Tokyo, Tokyo, Japan

${ }^{\ddagger}$ Present address: Los Alamos National Laboratory, Los Alamos, NM 87545, USA

${ }^{\S}$ Present address: School of Natural Sciences, Institute for Advanced Study, Princeton, NJ 08540, USA

"Present address: Kansas State University, Manhattan, KS 66506, USA

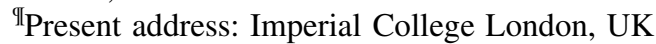

[1] KamLAND Collaboration, K. Eguchi et al., Phys. Rev. Lett. 90, 021802 (2003)

[2] L. Wolfenstein, Phys. Rev. D 17, 2369 (1978); S.P. Mikheyev and A. Yu. Smirnov, Sov. J. Nucl. Phys. 42, 913 (1986).

[3] ${ }^{235} \mathrm{U}$ : K. Schreckenbach et al., Phys. Lett. B 160, 325 (1985); ${ }^{239241} \mathrm{Pu}$ : A. A. Hahn et al., Phys. Lett. B 218, 365 (1989); ${ }^{238} \mathrm{U}$ : P. Vogel et al., Phys. Rev. C 24, 1543 (1981).

[4] K. K. Sekharan et al., Phys. Rev. 156, 1187 (1967); We actually used an R-matrix evaluation from http:// wwwndc.tokai.jaeri.go.jp/ftpnd/jendl/jendl-an-2003.html of this measured cross section.

[5] P. Vogel and J.F. Beacom, Phys. Rev. D 60, 053003 (1999); A. Kurylov et al., Phys. Rev. C 67, 035502 (2003).

[6] J. M. Herndon, Proc. Nat. Acad. Sci. U.S.A. 100, 3047 (2003).

[7] A. Stuart et al., Kendall's Advanced Theory of Statistics, (Oxford University Press, New York 1999), Vol. 2A.

[8] V. D. Barger et al., Phys. Rev. Lett. 82, 2640 (1999).

[9] E. Lisi et al., Phys. Rev. Lett. 85, 1166 (2000).

[10] J.F. Beacom and N.F. Bell, Phys. Rev. D 65, 113009 (2002).

[11] J. A. Frieman et al., Phys. Lett. B 200, 115 (1988); R. S. Raghavan et al., Phys. Rev. D 38, 1317 (1988).

[12] J. N. Bahcall and C. Peña-Garay, New J. Phys. 6, 63 (2004); M. Maltoni et al., New J. Phys. 6, 122 (2004).

[13] SNO Collaboration, S. N. Ahmed et al. Phys. Rev. Lett. 92, 181301 (2004). 